\title{
Improved description of the nucleon polarizabilities with relativistic Chiral Effective Field Theory
}

\author{
Jose Manuel Alarcón* \\ Helmholtz-Institut für Strahlen- und Kernphysik \& Bethe Center for Theoretical Physics, \\ Universität Bonn, 53115 Bonn, Germany \\ E-mail: alarcon@hiskp.uni-bonn.de
}

\begin{abstract}
In this contribution we present a calculation of the doble virtual Compton scattering process at low $Q^{2}$. We employ the relativistic formulation of chiral effective field theory with baryons, including also the $\Delta(1232)$ as a dynamical degree of freedom. The $Q^{2}$ evolution of the lowest order scalar and spin polarizabilities are extracted from the Compton tensor, and are compared to experimental as well as to previous chiral and dispersive determinations. We also discuss the impact of this improvement in the Proton Radius Puzzle.
\end{abstract}

The 8th International Workshop on Chiral Dynamics, CD2015 ***

29 June 2015 - 03 July 2015

Pisa,Italy

\footnotetext{
* Speaker.
} 


\section{Introduction}

The study of the internal electromagnetic structure of the nucleon has been a very active field since decades from both, theoretical and experimental sides. Several experiments have been conducted along these years with the final goal of understanding, from first principles, how the nucleon responds under electromagnetic probes. This will lead to a better determination of the output of experiments in which the electromagnetic response of the nucleon plays a prominent role. To the level of precision of the experimental programs running nowadays, one usually needs to go beyond the one photon exchange approximation. One important example is the study of the $e^{-} p$ scattering, where the two photon exchange (TPE) corrections can play a fundamental role in the determination of the proton radius [1]. These corrections have two kinds of contributions, one coming from the form factors of the nucleon, and another generated by the polarizabilities of the nucleon. This last one has been object of intensive study from the theory side, due to the potential that they have to solve the so-called "Proton Radius Puzzle" [2, 3, 4, 5, 6, 7, 8, 9, 10, 11, 12, 13, 14]. The polarizabilities are simply the moments of the electromagnetic response of the nucleon, and can be connected to their classical definition of through the low energy expansion of an effective $\gamma \gamma N N$ Lagrangian $[15,16]$. Their determination has been subject of several experimental programs in the past $[17,18,19,20,21,22,23,24]$, and are still object of recent experimental analyses.

Since the polarizabilities encode the response of the constituents of the nucleon under electromagnetic probes, they provide an excellent test ground for quantum chromodynamics (QCD). Unfortunately, in the energy region of interest here, QCD is not solvable through a perturbative approach, and the determination of the polarizabilities in terms of quark and gluon degrees of freedom becomes a tough task [25]. Nevertheless, it is still possible to work within a framework embodying the relevant symmetries of QCD at low energies, in which the degrees of freedom are not quarks and gluons but hadrons. This framework is commonly known as chiral effective field theory (chiral EFT), and has been very successful in explaining the structure of the nucleon on chiral symmetry grounds.

Here we will follow the relativistic approach [26, 27], including also the $\Delta(1232)$ resonance as a dynamical degree of freedom, to study the $Q^{2}$ evolution of the lowest order scalar and spin polarizabilities of the nucleon appearing in the double virtual Compton scattering (VVCS). Later, we also study the impact of the improved description of these polarizabilities on the calculation of the polarizability contribution to the muonic hydrogen Lamb shift.

\section{Polarizabilities}

The object of our study are the lowest-order polarizabilities entering in the description of the forward VVCS. Traditionally, the amplitude describing this process is written in the following non-relativistic form

$$
T\left(v, Q^{2}\right)=f_{L}\left(v, Q^{2}\right)+\left(\vec{\varepsilon}^{\prime} \cdot \vec{\varepsilon}\right) f_{T}\left(v, Q^{2}\right)+i \vec{\sigma} \cdot\left(\vec{\varepsilon}^{\prime} * \vec{\varepsilon}\right) g_{T T}\left(v, Q^{2}\right)-i \vec{\sigma} \cdot\left[\left(\vec{\varepsilon}^{\prime} * \varepsilon\right) \times \hat{q}\right] g_{L T}\left(v, Q^{2}\right)
$$

where $v=\left(s-m_{N}^{2}+Q^{2}\right) / 2 m_{N}$ and $Q^{2}$ are the photon energy and virtuality, respectively, being $m_{N}$ the nucleon mass, and $\vec{q}$ the three momentum of the photon. Notice that $f_{L}$ and $f_{T}$ encode the 

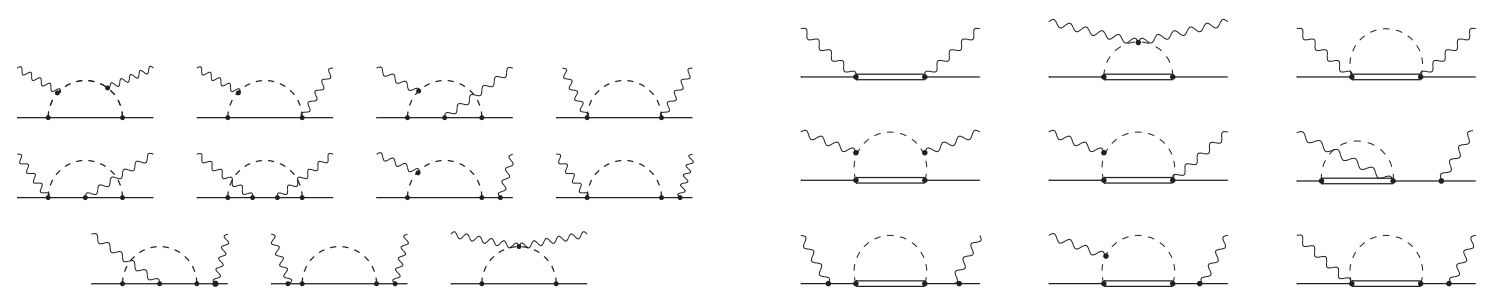

Figure 1: Diagrams computed in the evaluation of the Compton tensor. The left panel shows the diagrams with the $\pi N$ loops, while the right one shows the diagrams that involve the $\Delta$ as intermediate state.

spin-independent response of the nucleon, while $g_{T T}$ and $g_{L T}$ contain information about the spindependent one. These functions can be splitted into a Born and a non-Born part. While the Born part is determined by the global properties of the nucleon as the mass, charge and anomalous magnetic moment $[28,29]$, the non-Born part is related to the polarizabilities. A low energy expansion of these functions allows us to identify the polarizabilities as the coefficients of the expansions in terms of $v$ and $Q^{2}$

$$
\begin{aligned}
& f_{T}\left(v, Q^{2}\right)=f_{T}^{(B o r n)}\left(v, Q^{2}\right)+4 \pi Q^{2} \beta_{M 1}+4 \pi\left(\alpha_{E 1}+\beta_{M 1}\right) v^{2}+\ldots \\
& f_{L}\left(v, Q^{2}\right)=f_{L}^{(B o r n)}\left(v, Q^{2}\right)+4 \pi \alpha_{E 1} Q^{2}+4 \pi \alpha_{L} v^{2} Q^{2}+\ldots \\
& g_{T T}\left(v, Q^{2}\right)=g_{T T}^{(B o r n)}\left(v, Q^{2}\right)+4 \pi \gamma_{0} v^{3}+\ldots \\
& g_{L T}\left(v, Q^{2}\right)=g_{L T}^{(B o r n)}\left(v, Q^{2}\right)+4 \pi \delta_{L T} v^{2} Q+\ldots
\end{aligned}
$$

As commented in the introduction, the approach that we use to calculate these functions is the relativistic formulation of chiral EFT with baryons, including the $\Delta(1232)$-resonance as an explicit degree of freedom. This approach has provided important progress in the understanding of fundamental hadronic reactions involving one baryon, as well as on the structure of the nucleon $[30,31,32,33,34,35,36]$. Here we perform the calculation up to order $\mathscr{O}\left(p^{4} / \Delta\right)$ in the $\delta$-counting. The diagrams inlvolved in this calculation are shown in Fig. 1. Up to this order, the calculation is a prediction, i. e., there is no free parameter to fit. In our calculations, we also include a dipole form factor in the magnetic $\gamma N \Delta$ transition form factor

$$
g_{M} \rightarrow \frac{g_{M}}{\left(1+Q^{2} / 0.71\right)^{2}}
$$

following Ref. [37]. There, it was shown that this inclusion is important in order to agree with data on electroproduction, which is related, via dispersion relations, to the $Q^{2}$ evolution of the polarizabilities.

In Fig. 2 we show our results (blue band) for the scalar polarizabilities of the proton and neutron. These are compared to the leading-order heavy baryon (HB) result (blue dashed line) and the MAID model (black dotted line). We also plot the leading-order relativistic result (red line) to show importance of the $\Delta(1232)$. In this figure, as well as in Fig. 3, the error bands (the blue ones) are calculated using a relative $Q^{2}$-dependent error $\tilde{\delta}\left(Q^{2}\right)$ defined as: 


$$
\tilde{\delta}\left(Q^{2}\right)=\frac{1}{3}\left(\frac{\delta}{m_{N}}+\sqrt{\frac{M_{\pi}}{m_{N}}}+\sqrt{\frac{Q^{2}}{m_{N}^{2}}}\right)
$$

for $Q<\delta$, and

$$
\tilde{\delta}\left(Q^{2}\right)=\sqrt{\frac{Q^{2}}{\delta^{2}}}
$$

for $Q>\delta$, being $M_{\pi}$ and $m_{\Delta}$ the pion and $\Delta(1232)$ masses, respectively, and $\delta=m_{\Delta}-m_{N}$.

We see in this figure that, in fact, it is very important in the $Q^{2}$ dependence of the combination $\alpha_{E 1}+\beta_{M 1}$, specially at low $Q^{2}$. This is not surprising, since we know that this resonance plays a very important role in $\beta_{M 1}$. On the other hand, regarding $\alpha_{L}$, we see that the leading order is enough, in a relativistic calculation, to reproduce very well the $Q^{2}$ evolution of this polarizability for both, the proton and the neutron. Notice that this is not the case in the HB approach. Therefore, the faster convergence of the relativistic approach makes an important difference in this polarizability.
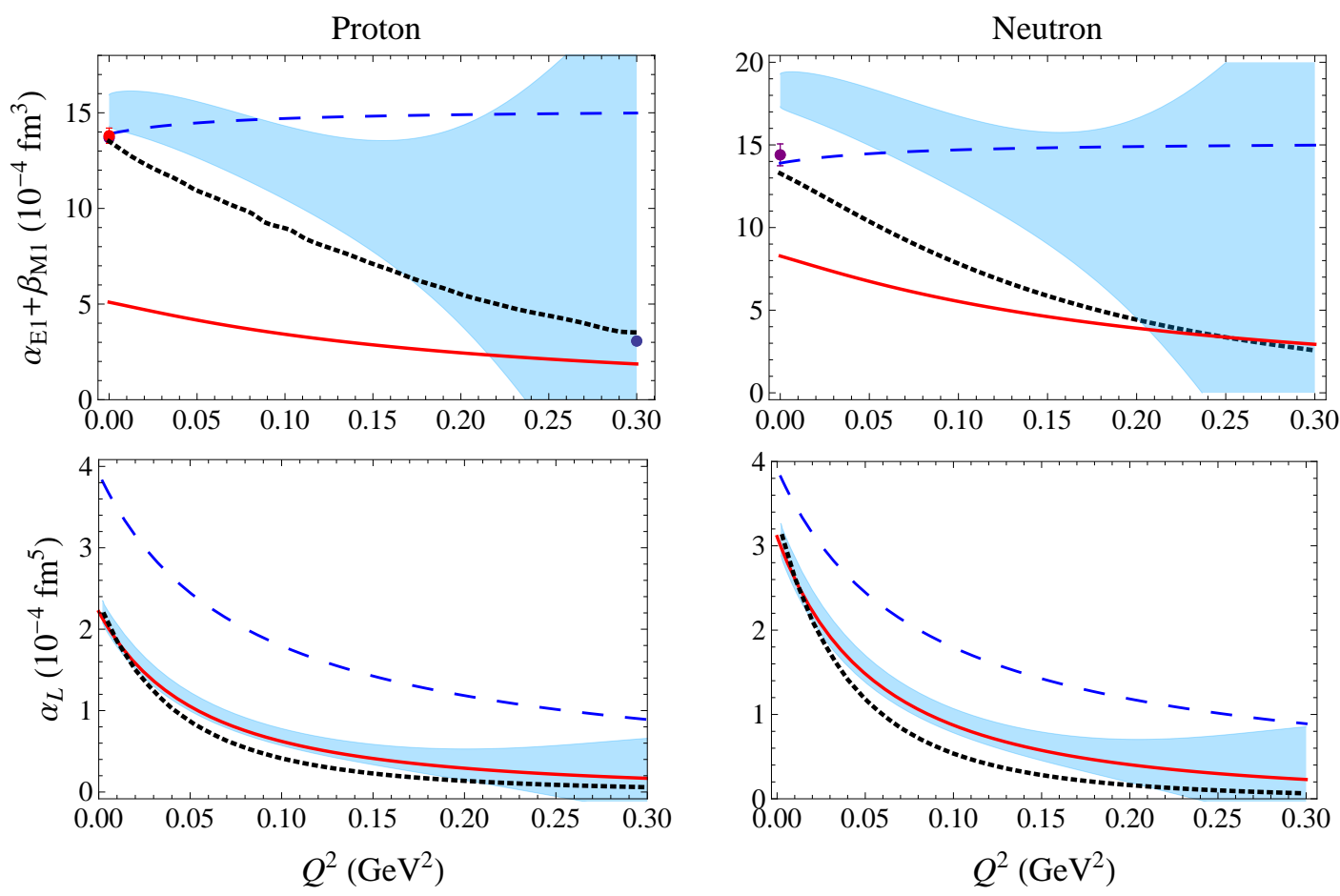

Figure 2: Results for the scalar polarizabilities. The red solid lines and blue bands represent, respectively, the LO and the full result (with $\Delta$ ) obtained in our calculation. The blue dashed line is the LO result in the HB limit. The black dotted lines represents the empirical result of MAID [38]. The data points at $Q^{2}=0$ correspond to Refs [22] and [17] (red and purple point, respectively) for the proton, and [17] for the neutron. The data point in the left upper panel at $Q^{2}=0.3 \mathrm{GeV}^{2}$ is from Ref. [18] .

In the case of the spin polarizabilities, Fig. 3, one sees that the complete result (with $\Delta$ ) agrees, in general, quite well with the MAID model and the experimental determinations. We also see the importance of the $\Delta(1232)$ in the $Q^{2}$ evolution of the forward-spin polarizability. Once this resonance is included in the calculation, the relativistic approach achieves a better description than the 
infrared (red band) or heavy baryon one (out of the range of the plot). Regarding $\delta_{L T}$ one sees that the leading order relativistic calculation already achieves a good description of this polarizability in the range of $Q^{2}$ considered. Once the $\Delta(1232)$ is included, the final result tends to the experimental as well as to the MAID results. Notice that Ref. [34] already calculated these polarizabilities at $\mathscr{O}\left(\varepsilon^{3}\right)$ in the relativistic approach (grey band). While their result agrees with ours for $\delta_{L T}^{n}$, it is very different for $\delta_{L T}^{p}$. This difference can be traced back to the diagrams in which the two photons couple to an internal delta propagator [39], which is of higher order in our counting. We expect to study the impact of these higher oder contributions in $\delta_{L T}^{p}$ in the future. In Table 1 we summarize the results at the real photon point, and compare them with the experimental determinations or the MAID model results.
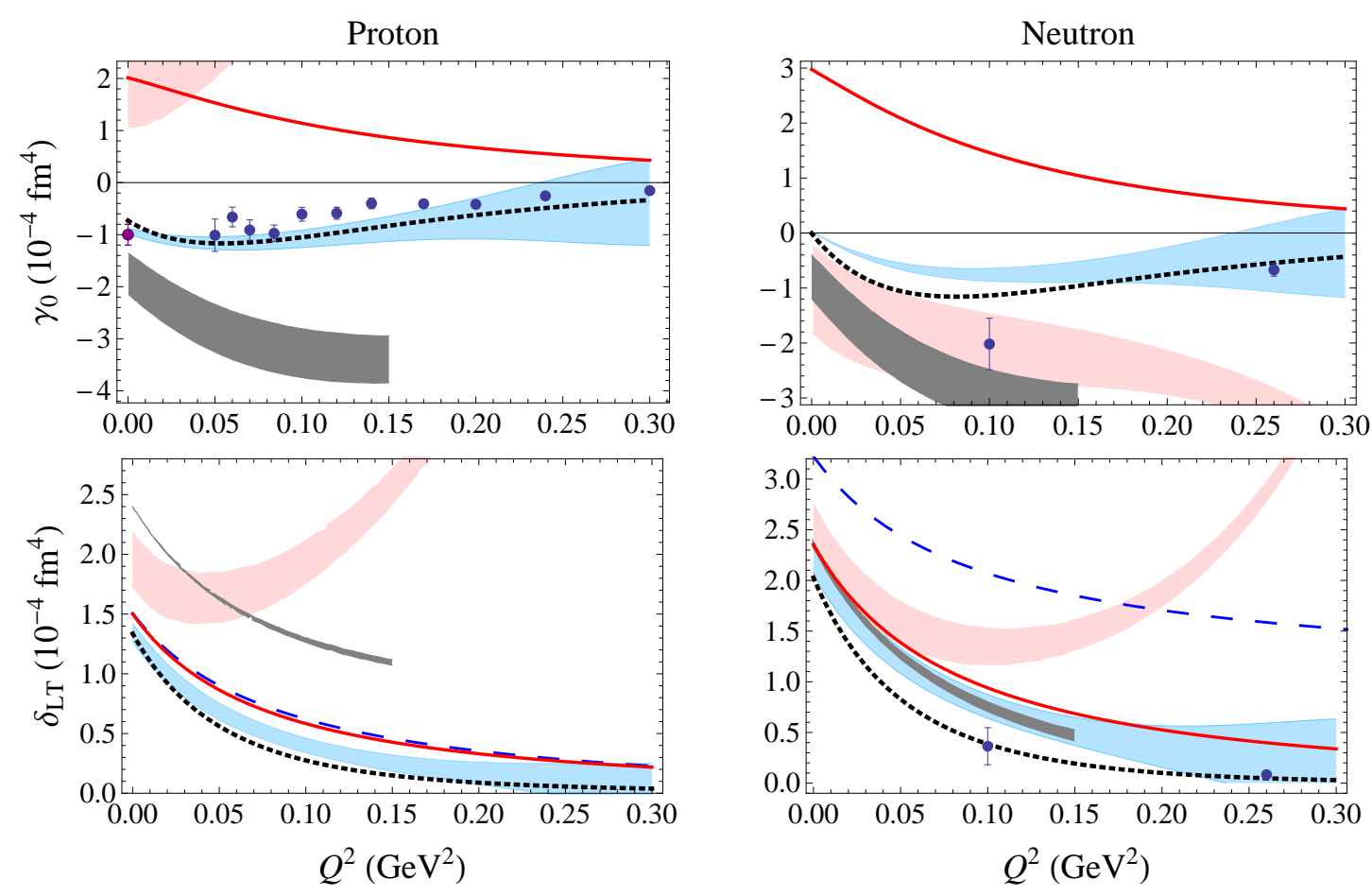

Figure 3: Results for the spin polarizabilities. The red solid lines and blue bands represent, respectively, the LO and the full result (with $\Delta$ ) that we obtained. Black dotted lines represent the MAID results [38]. The grey bands are the relativistic calculation of Ref. [34]. The blue dashed line is the $\mathscr{O}\left(p^{4}\right) \mathrm{HB}$ calculation [42]; off the scale in the upper panels. The light-red band is the IR calculation [43]. The data points for the proton $\gamma_{0}$ at finite $Q^{2}$ are from Ref. [19] (blue dots), and at $Q^{2}=0$ from [20] (purple square). For the neutron all the data are from Ref. [21].

\section{The Lamb shift and the Proton Radius Puzzle}

These improvements in the description of the $Q^{2}$ evolution of the polarizabilities have important consequences in searches of physics beyond the standard model. To be specific, they intervene in the extraction of the proton radius through the measurement of the Lamb shift. Although for the normal hydrogen this contribution is negligible, it can play a relevant role in the case of muonic 


\begin{tabular}{|c|c|c|c|c|}
\hline & \multicolumn{2}{|c|}{ Proton } & \multicolumn{2}{|c|}{ Neutron } \\
\hline & $\begin{array}{l}\text { Our work } \\
\text { Ref. [40] }\end{array}$ & Empirical & $\begin{array}{l}\text { Our work } \\
\text { Ref. [40] }\end{array}$ & Empirical \\
\hline $\begin{array}{l}\alpha_{E 1}+\beta_{M 1} \\
\left(10^{-4} \mathrm{fm}^{3}\right)\end{array}$ & $15.12(82)$ & $\begin{array}{c}13.8(4) \\
\text { Ref. [22] }\end{array}$ & $18.30(99)$ & $\begin{array}{l}14.40(66) \\
\text { Ref. [17] }\end{array}$ \\
\hline $\begin{array}{c}\alpha_{L} \\
\left(10^{-4} \mathrm{fm}^{5}\right)\end{array}$ & $2.31(12)$ & $\begin{array}{c}2.32 \\
\text { [MAID] }\end{array}$ & $3.21(17)$ & $\begin{array}{c}3.32 \\
\text { [MAID] }\end{array}$ \\
\hline $\begin{array}{c}\gamma_{0} \\
\left(10^{-4} \mathrm{fm}^{4}\right)\end{array}$ & $-0.93(5)$ & $\begin{array}{c}-1.00(8)(12) \\
\text { Ref. [20] }\end{array}$ & $0.05(1)$ & $\begin{array}{l}-0.005 \\
{[\mathrm{MAID}]}\end{array}$ \\
\hline $\begin{array}{c}\delta_{L T} \\
\left(10^{-4} \mathrm{fm}^{4}\right)\end{array}$ & $1.35(7)$ & $\begin{array}{c}1.34 \\
\text { [MAID] }\end{array}$ & $2.20(12)$ & $\begin{array}{c}2.03 \\
\text { [MAID] }\end{array}$ \\
\hline
\end{tabular}

Table 1: Predictions for the forward VVCS polarizabilities at the real photon point compared with the available empirical information. Where [MAID] is shown, the empirical number is provided by the MAID analysis [41,38].

hydrogen $(\mu H)$, since the muon is much closer to the proton in this case, and it is much more sensitive to its internal structure. In fact, the particular features of the polarizabilities contribution to the Lamb shift make it a suitable candidate to solve the so-called "Proton Radius Puzzle". This puzzle emerges from the discrepancy between the Lamb shift measured in $\mu H$ and the expected value based on the proton radius reported by CODATA in 2010 [44],

$$
\Delta E_{2 P-2 S}^{e x p}-\Delta E_{2 P-2 S}^{t h}\left(r_{E}^{\mathrm{CODATA}}\right)=310 \mu \mathrm{eV}
$$

which has a statistical significance of about $7 \sigma$. This difference has been attributed to a mismatch between the radius deduced in the $\mu H$ Lamb shift measurements compared to both, the electron-proton scattering and normal hydrogen Lamb shift determinations. However, it is important to stress that recent extractions of the proton radius from modern measurements of electron-proton scattering [45] using physically constrained form factors (that incorporate analyticity, causality and crossing symmetry) reconcile the electron-proton scattering and the $\mu H$ Lamb shift determination of the proton radius [46, 47, 48], reducing the discrepancy to $4 \sigma$.

One possible missing (or underestimated) piece of information could come from the polarizabilities of the proton. In Fig. 4 we show the two-photon exchange corrections to which the polarizabilities contribute.

The polarizabilities contribution starts at $\mathscr{O}\left(\alpha_{e m}^{5}\right)$. At this order, one can consider forward kinematics for the Compton subprocess, since off-forward contributions are suppressed by $\alpha_{e m}$. This allows us to use the results of the previous section to compute the leading-order prediction of the polarizabilities contribution to the $\mu H$ Lamb shift. It is simple to show that the effect of this correction to the Lamb shift can be well approximated by (see Ref. [11])

$$
\Delta E_{2 S}^{p o l} \approx \frac{\alpha_{e m}}{\pi} \phi_{n=2}^{2} \int_{0}^{\infty} \frac{d Q}{Q^{2}} w\left(\frac{Q^{2}}{4 m_{\ell}^{2}}\right)\left[T_{1}^{(N B)}\left(0, Q^{2}\right)-T_{2}^{(N B)}\left(0, Q^{2}\right)\right], \quad w(x)=\sqrt{1+x}-\sqrt{x}
$$




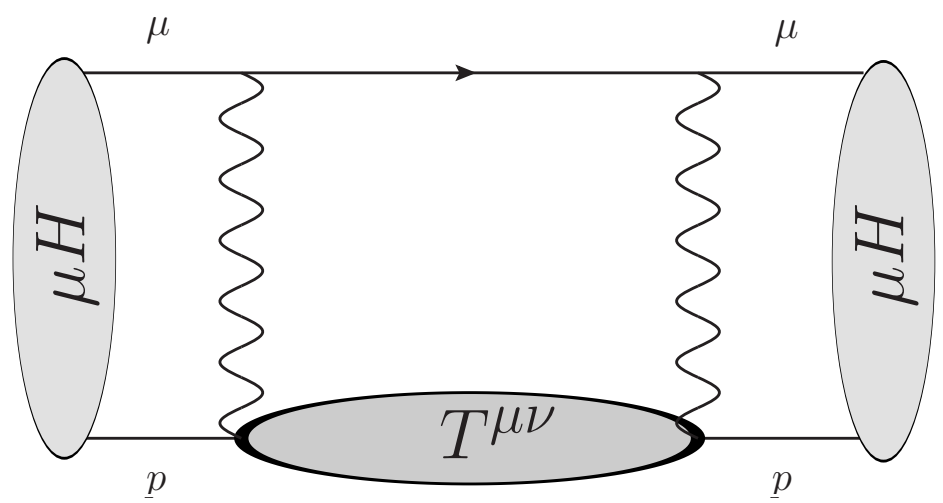

Figure 4: Two photon exchange corrections to the $\mu H$. The $T^{\mu v}$ symbolizes the Compton tensor.

\begin{tabular}{ccccccccc}
\hline & & Marty- & Nevado \& & Carlson \& & Birse \& & Gorchtein & This & Peset \\
et al. & work & \& Pineda \\
$(\mu \mathrm{eV})$ & {$[4]$} & {$[5]$} & {$[6]$} & {$[7]$} & {$[9]$} & {$[10]$} & {$[11]$} & {$[12]$} \\
\hline$\Delta E_{2 S}^{(\mathrm{pol})}$ & $-12(2)$ & -11.5 & -18.5 & $-7.4(2.4)$ & $-8.5(1.1)$ & $-15.3(5.6)$ & $-8.2\left({ }_{-2.5}^{+1.2}\right)$ & -26.5 \\
\hline
\end{tabular}

Table 2: Summary of the different determinations of the polarizability correction to the $\mu H$ Lamb shift.

where $\phi_{n=2}$ is the wave function of the $\mu H$ at the origin, $m_{\ell}$ is the mass of the lepton (muon), and $T_{1}^{(N B)}$ and $T_{2}^{(N B)}$ stand for the non-Born part of $T_{1}$ and $T_{2}$, defined from the spin-independent part of the Compton tensor in the following way

$$
T^{\mu v}(P, q)=\left(-g^{\mu v}+\frac{q^{\mu} q^{v}}{q^{2}}\right) T_{1}\left(v, Q^{2}\right)+\frac{1}{M_{N}^{2}}\left(P^{\mu}-\frac{P \cdot q}{q^{2}} q^{\mu}\right)\left(P^{v}-\frac{p \cdot q}{q^{2}} q^{v}\right) T_{2}\left(v, Q^{2}\right) .
$$

Even though this integral extends to regions of $Q^{2}$ to which chiral EFT is not applicable, it is important to notice that, for high $Q^{2}$, the contribution of $T_{1}$ and $T_{2}$ is suppressed by the weighting function $w\left(\tau_{\ell}\right)$. In fact, in Ref. [11] it was proved that this integral converges quickly to its asymptotic value (i. e., when the upper limit $Q_{\max }^{2}=\infty$ ) in the low $Q^{2}$ region when using the relativistic approach. However, this is not the case for the heavy baryon formulation, that exhibits a much slower convergence. This is shown in Fig. 5 by plotting the relativistic and HB results as a function of $Q_{\max }^{2}$. While at $Q^{2} \sim 0.2 \mathrm{GeV}^{2}$ already the relativistic approach gives a result compatible, within the expected systematic error, with the asymptotic value, the HB one does not, even at large $Q^{2}\left(\sim 1 \mathrm{GeV}^{2}\right)$.

In Table 2 we summarize the results for $\Delta E_{2 S}^{(\mathrm{pol})}$ available in the literature. Most of them are based on dispersive approaches, that employ experimental information. Only [6], [11], [12] are chiral predictions. Among them, only the relativistic approach gives a result, $\Delta E_{2 S}^{(\mathrm{pol})}=-8.2\left({ }_{-2.5}^{+1.2}\right) \mu \mathrm{eV}$ [11], compatible with the preferred value nowadays, $\Delta E_{2 S}^{(\mathrm{pol})}=-8.5(1.1) \mu \mathrm{eV}$ [7, 9]. Although this contribution is one order of magnitude smaller than required to explain the Proton Radius Puzzle, this beautiful agreement between the chiral prediction and phenomenological extractions helps in establishing the size and uncertainty of $\Delta E_{2 S}^{(\mathrm{pol})}$ on EFT grounds. 


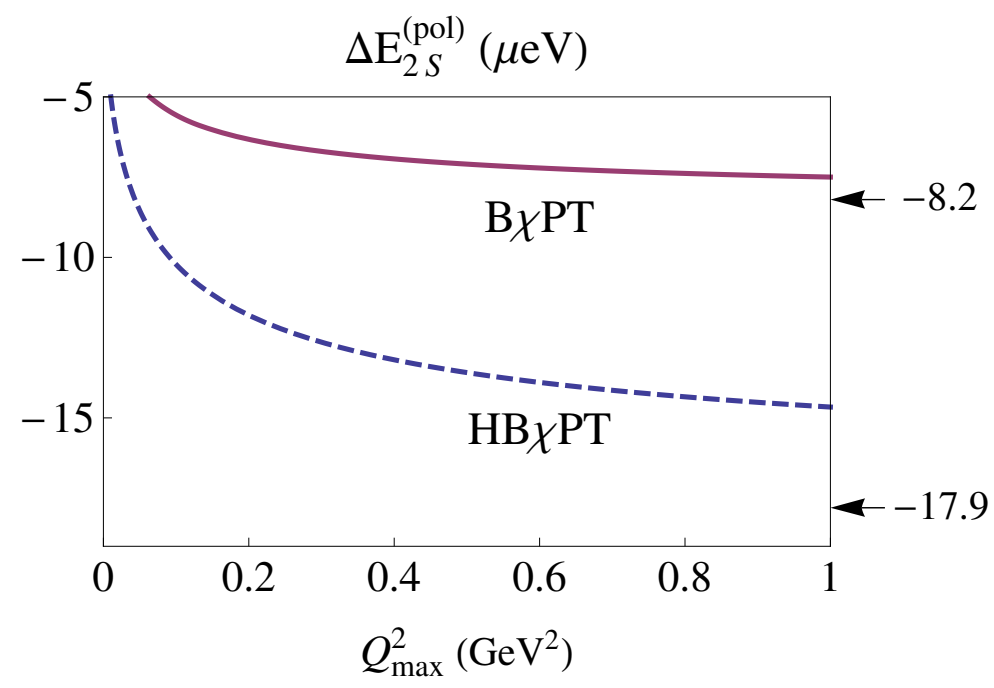

Figure 5: Result of the integral in Eq. (3.2) as a function of its upper limit, $Q_{\max }^{2}$.

\section{Summary and conclusions}

We have shown that a relativistic formulation of chiral EFT for baryons with $\Delta$ degrees of freedom achieves a better description of the lowest order scalar and spin polarizabilities of the nucleon at low $Q^{2}$. These improvements are important in order to understand the properties of the nucleon on QCD grounds, as well as for experimental investigations of the spin structure of the nucleon [49]. Our better knowledge of the electromagnetic response of the nucleon is also relevant in experimental searches of physics beyond the standard model in the low-energy frontier. Here we have shown how the Compton tensor calculated to extract the polarizabilities, also leads to a value of $\Delta E_{2 S}^{(\mathrm{pol})}$ that turns out to be in excellent agreement with the phenomenological extractions [7, 9], that are mostly based on experimental information. This agreement helps to establish the contribution of $\Delta E_{2 S}^{(\mathrm{pol})}$ to the measurement of the proton radius on chiral symmetry grounds.

\section{References}

[1] A. Antognini, F. Kottmann, F. Biraben, P. Indelicato, F. Nez and R. Pohl, Theory of the 2S-2P Lamb shift and 2S hyperfine splitting in muonic hydrogen, Annals Phys. 331, 127 (2013).

[2] R. Pohl, R. Gilman, G. A. Miller and K. Pachucki, Muonic hydrogen and the proton radius puzzle, Ann. Rev. Nucl. Part. Sci. 63, 175 (2013).

[3] G. A. Miller, Proton Polarizability Contribution: Muonic Hydrogen Lamb Shift and Elastic Scattering, Phys. Lett. B 718, 1078 (2013).

[4] K. Pachucki, Proton structure effects in muonic hydrogen, Phys. Rev. A 60, 3593 (1999).

[5] A. P. Martynenko, Proton polarizability effect in the Lamb shift of the hydrogen atom, Phys. Atom. Nucl. 69, 1309 (2006). 
[6] D. Nevado and A. Pineda, Forward virtual Compton scattering and the Lamb shift in chiral perturbation theory, Phys. Rev. C 77, 035202 (2008).

[7] C. E. Carlson and M. Vanderhaeghen, Higher order proton structure corrections to the Lamb shift in muonic hydrogen, Phys. Rev. A 84, 020102 (2011).

[8] R. J. Hill and G. Paz, Model independent analysis of proton structure for hydrogenic bound states, Phys. Rev. Lett. 107, 160402 (2011).

[9] M. C. Birse and J. A. McGovern, Proton polarisability contribution to the Lamb shift in muonic hydrogen at fourth order in chiral perturbation theory, Eur. Phys. J. A 48, 120 (2012).

[10] M. Gorchtein, F. J. Llanes-Estrada and A. P. Szczepaniak, $\mu$ - H Lamb shift: dispersing the nucleon-excitation uncertainty with a finite energy sum rule, Phys. Rev. A 87, 052501 (2013).

[11] J. M. Alarcon, V. Lensky and V. Pascalutsa, Chiral perturbation theory of muonic hydrogen Lamb shift: polarizability contribution, Eur. Phys. J. C 74, 2852 (2014).

[12] C. Peset and A. Pineda, Model independent determination of the muonic hydrogen Lamb shift and proton radius, Eur. Phys. J. A 51, no. 3, 32 (2015).

[13] C. Peset and A. Pineda, The two-photon exchange contribution to muonic hydrogen from chiral perturbation theory, Nucl. Phys. B 887, 69 (2014).

[14] C. Peset and A. Pineda, The Lamb shift in muonic hydrogen and the proton radius from effective field theories, Eur. Phys. J. A 51, no. 12, 156 (2015).

[15] H. W. Griesshammer, J. A. McGovern, D. R. Phillips and G. Feldman, Using effective field theory to analyse low-energy Compton scattering data from protons and light nuclei, Prog. Part. Nucl. Phys. 67, 841 (2012).

[16] N. Krupina and V. Pascalutsa, Separation of proton polarizabilities with the beam asymmetry of Compton scattering, Phys. Rev. Lett. 110, no. 26, 262001 (2013).

[17] D. Babusci, G. Giordano and G. Matone, A New evaluation of the Baldin sum rule, Phys. Rev. C 57, 291 (1998).

[18] Y. Liang, M. E. Christy, R. Ent and C. E. Keppel, $Q^{* * 2}$ evolution of generalized Baldin sum rule for the proton, Phys. Rev. C 73, 065201 (2006).

[19] Y. Prok et al. [CLAS Collaboration], Moments of the Spin Structure Functions $g^{* *} p(1)$ and $g * * d(1)$ for $0.05<Q^{* * 2}<3.0-G e V^{* * 2}$, Phys. Lett. B 672, 12 (2009).

[20] H. Dutz et al. [GDH Collaboration], First measurement of the Gerasimov-Drell-Hearn sum rule for H-1 from 0.7-GeV to 1.8-GeV at ELSA, Phys. Rev. Lett. 91, 192001 (2003).

[21] M. Amarian et al. [Jefferson Lab E94010 Collaboration], Measurement of the generalized forward spin polarizabilities of the neutron, Phys. Rev. Lett. 93, 152301 (2004).

[22] V. Olmos de Leon, F. Wissmann, P. Achenbach, J. Ahrens, H. J. Arends, R. Beck, P. D. Harty and V. Hejny et al., Low - energy Compton scattering and the polarizabilities of the proton, Eur. Phys. J. A 10, 207 (2001).

[23] M. Amarian, L. Auerbach, T. Averett, J. Berthot, P. Bertin, W. Bertozzi, T. Black and E. Brash et al., The $Q^{* * 2}$ evolution of the generalized Gerasimov-Drell-Hearn integral for the neutron using a He-3 target, Phys. Rev. Lett. 89, 242301 (2002).

[24] M. Amarian et al. [Jefferson Lab E94-010 Collaboration], $Q * * 2$ evolution of the neutron spin structure moments using a He-3 target, Phys. Rev. Lett. 92, 022301 (2004). 
[25] M. Lujan, A. Alexandru, W. Freeman and F. Lee, Electric polarizability of neutral hadrons from dynamical lattice QCD ensembles, Phys. Rev. D 89, 074506 (2014).

[26] T. Fuchs, J. Gegelia, G. Japaridze and S. Scherer, Renormalization of relativistic baryon chiral perturbation theory and power counting, Phys. Rev. D 68, 056005 (2003).

[27] J. Gegelia and G. Japaridze, Matching heavy particle approach to relativistic theory, Phys. Rev. D 60, 114038 (1999).

[28] F. E. Low, Scattering of light of very low frequency by systems of spin 1/2, Phys. Rev. 96, 1428 (1954).

[29] M. Gell-Mann and M. L. Goldberger, Scattering of low-energy photons by particles of spin 1/2 Phys. Rev. 96, 1433 (1954).

[30] V. Lensky and V. Pascalutsa, Predictive powers of chiral perturbation theory in Compton scattering off protons, Eur. Phys. J. C 65, 195 (2010).

[31] J. M. Alarcon, J. Martin Camalich and J. A. Oller, The chiral representation of the $\pi N$ scattering amplitude and the pion-nucleon sigma term, Phys. Rev. D 85, 051503 (2012).

[32] J. M. Alarcon, J. Martin Camalich and J. A. Oller, Improved description of the $\pi N$-scattering phenomenology in covariant baryon chiral perturbation theory, Annals Phys. 336, 413 (2013).

[33] J. M. Alarcon, L. S. Geng, J. Martin Camalich and J. A. Oller, The strangeness content of the nucleon from effective field theory and phenomenology, Phys. Lett. B 730, 342 (2014).

[34] V. Bernard, E. Epelbaum, H. Krebs and U. G. Meißner, New insights into the spin structure of the nucleon, Phys. Rev. D 87, no. 5, 054032 (2013).

[35] A. N. Hiller Blin, T. Ledwig and M. J. Vicente Vacas, Chiral dynamics in the $\vec{\gamma} p \rightarrow p \pi^{0}$ reaction, Phys. Lett. B 747, 217 (2015).

[36] A. H. Blin, M. V. Vacas and T. Ledwig, Threshold Neutral Pion Photoproduction on the Proton, arXiv:1508.03486 [hep-ph].

[37] V. Pascalutsa and M. Vanderhaeghen, Phys. Rev. D 73, 034003 (2006).

[38] D. Drechsel, S. S. Kamalov and L. Tiator, Unitary Isobar Model - MAID2007, Eur. Phys. J. A 34, 69 (2007).

[39] H. Krebs, Private communication.

[40] V. Lensky, J. M. Alarcón and V. Pascalutsa, Moments of nucleon structure functions at next-to-leading order in baryon chiral perturbation theory, Phys. Rev. C 90, no. 5, 055202 (2014).

[41] D. Drechsel, S. S. Kamalov and L. Tiator, The GDH sum rule and related integrals, Phys. Rev. D 63, 114010 (2001).

[42] C. W. Kao, T. Spitzenberg and M. Vanderhaeghen, Burkhardt-Cottingham sum rule and forward spin polarizabilities in heavy baryon chiral perturbation theory, Phys. Rev. D 67, 016001 (2003).

[43] V. Bernard, T. R. Hemmert and U. -G. Meißner, Spin structure of the nucleon at low-energies, Phys. Rev. D 67, 076008 (2003).

[44] A. Antognini, F. Kottmann, F. Biraben, P. Indelicato, F. Nez and R. Pohl, Theory of the 2S-2P Lamb shift and 2 S hyperfine splitting in muonic hydrogen, Annals Phys. 331, 127 (2013).

[45] J. C. Bernauer et al. [A1 Collaboration], Phys. Rev. Lett. 105, 242001 (2010); 
[46] I. T. Lorenz, H.-W. Hammer and U.-G. Meißner, The size of the proton - closing in on the radius puzzle, Eur. Phys. J. A 48, 151 (2012).

[47] I. T. Lorenz and U.-G. Meißner, Reduction of the proton radius discrepancy by $3 \sigma$, Phys. Lett. B 737, 57 (2014).

[48] I. T. Lorenz, U.-G. Meißner, H.-W. Hammer and Y.-B. Dong, Theoretical Constraints and Systematic Effects in the Determination of the Proton Form Factors, Phys. Rev. D 91, no. 1, 014023 (2015).

[49] K. Allada An overview of recent nucleon spin structure measurements at Jefferson Lab. Plenary talk presented in this workshop. 